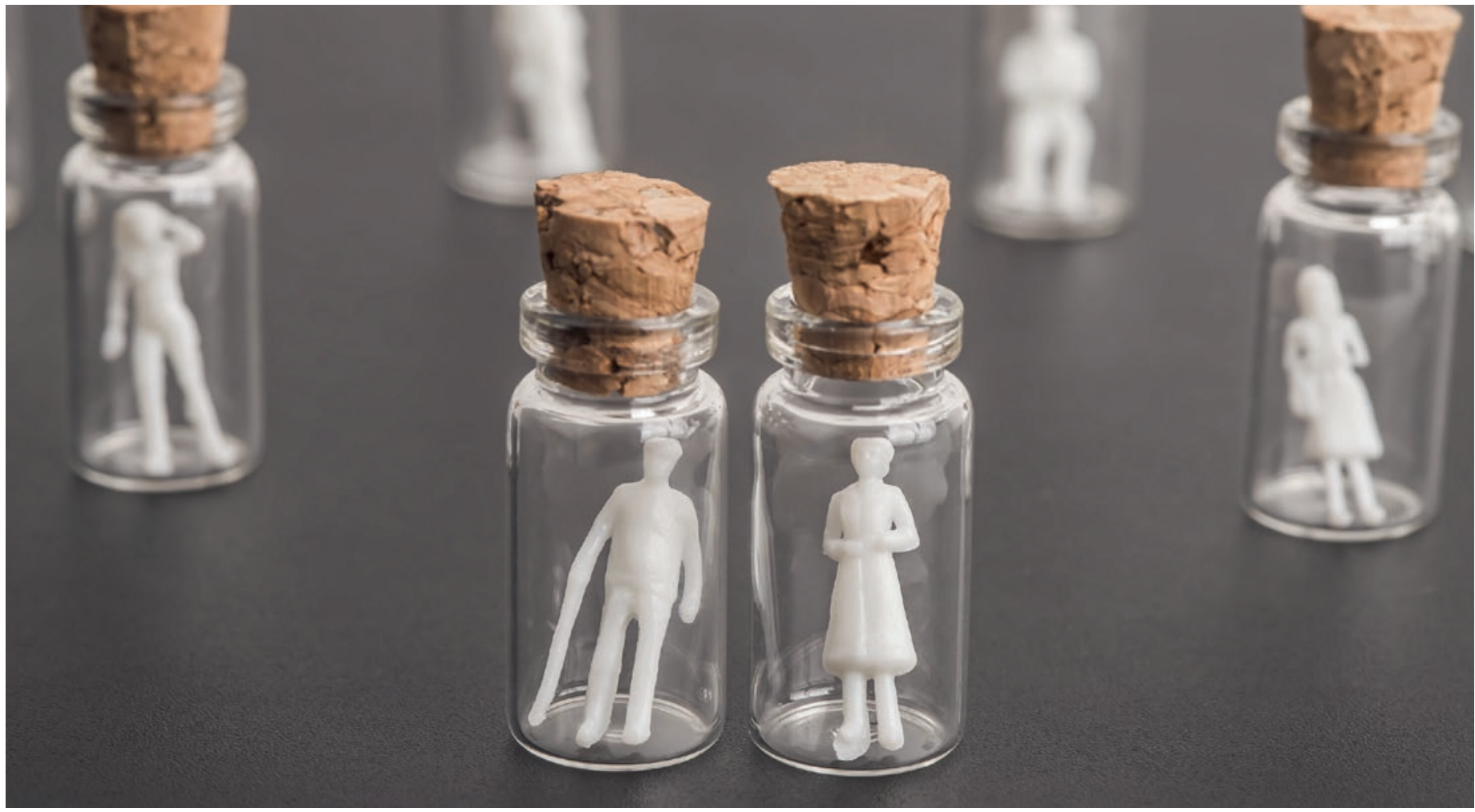

Der Schutz, das Wohlergehen und der Erhalt der Lebensqualität von vulnerablen Personen sind eine gesellschaftliche Pflicht.

\title{
Solidarität und Verantwortung in der Pandemie
}

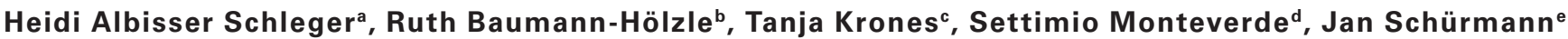

${ }^{a}$ Dr. phil., Medizinethikerin, Universität Basel, Institut für Pflegewissenschaft; ${ }^{b}$ Dr. theol., Medizinethikerin, Institut Dialog Ethik; ${ }^{c}$ Prof. Dr. med., Dipl. Soz., Klinische Ethikerin, Universität Zürich, Institut für Biomedizinische Ethik und Medizingeschichte, Universitätsspital Zürich; d Prof. (FH), PhD, RN, Klinischer Ethiker, Hochschuldozent, Berner Fachhochschule, Departement Gesundheit, Universität Zürich, Institut für Biomedizinische Ethik und Medizingeschichte, Universitätsspital Zürich; e MA, Klinischer Ethiker, Universitätsspital Basel

Die Corona-Pandemie berührt weite Bereiche des gesellschaftlichen, wirtschaftlichen und politischen Lebens. Mit hoher Dringlichkeit stellt sich angesichts der Ressourcenknappheit die Forderung nach einer konkret gelebten Solidarität und verantwortungsvollen Koordination zwischen Behörden, Organisationen, Institutionen, Generationen und Individuen. Medizinethikerinnen und -ethiker der Schweiz formulieren dazu vier Postulate.

In der ersten Pandemiewelle standen die drohende Überlastung des Gesundheitswesens, der Schutz des Lebens und der Lebensqualität besonders vulnerabler Personengruppen und die Ausstattung von Gesundheitseinrichtungen mit Schutzmaterial im Vordergrund. Die Schweizerische Akademie der Medizinischen Wissenschaften (SAMW) [1], die Schweizerische Gesellschaft für Intensivmedizin (SGI) [1, 2], die Nationale Ethikkommission im Bereich der Humanmedizin
[3], die Swiss National COVID-19 Science Task Force [4], der Schweizerische Berufsverband der Pflegefachfrauen und der Pflegefachmänner [5-7], die Stiftung Dialog Ethik [8] sowie eine Gruppe von Medizinethikerinnen und Medizinethikern der Schweiz [9] haben wiederholt auf damit verbundene ethische Herausforderungen hingewiesen. In der zweite Pandemiewelle haben sich diese Herausforderungen verschärft, es sind aber auch weitere hinzugekommen. So zeigen 
sich neue Personengruppen, die für die Auswirkungen der Pandemie besonders verletzlich sind, wie etwa Kinder, Familien, Alleinerziehende, alleinstehende Seniorinnen und Senioren oder Menschen in prekären sozialen Lagen. Zudem werden durch die CoronaPandemie existierende Herausforderungen im Gesundheitswesen verschärft: Unterfinanzierung von ambulanten und stationären Leistungen, fehlende Finanzierung von Pandemie-spezifischen Massnah-

\section{Die Grundversorgung muss gestärkt und verletzliche Personengruppen müssen ge- schützt werden.}

men, mangelnde Koordination der Leistungserbringer im Umgang mit knappen Ressourcen, aber auch Fachkräftemangel sowie die physische und psychische Erschöpfung des Gesundheitspersonals. Diese schon bekannten Herausforderungen bedrohen in der Corona-Pandemie den Sicherstellungsauftrag von Institutionen der Gesundheitsversorgung für die gesamte Wohnbevölkerung.

\section{Vier Postulate}

Die Autorinnen und Autoren des vorliegenden Appells zeigen im Folgenden vier Aspekte auf, in denen sie das ethische Postulat einer gelebten Solidarität und Verantwortung besonders gefährdet sehen. Sie fordern die Verantwortlichen auf der Ebene von Bund, Kantonen, Gemeinden, Leistungserbringern und -finanzierern auf, rasch konkrete Massnahmen zu ergreifen, welche die Grundversorgung stärken, besonders verletzliche Personengruppen schützen, die Transparenz in der Koordination von Verlegungen sowie der Triage, sofern diese unvermeidlich ist, sicherstellen .

\section{Stärkung der Grundversorgung}

Die medizinische Grundversorgung ist der Grundpfeiler eines funktionsfähigen Gesundheitswesens. Diese muss gerade auch in der Pandemie gestärkt werden. Dies schliesst die verstärkte Einbindung der Hausarztmedizin und der Spitex-Dienste in die öffentlichen Gesundheitsmassnahmen zur Bekämpfung der Pandemie ein sowie die häusliche Betreuung und Versorgung von COVID-19- und anderen schwer Erkrankten, die keine Hospitalisation wünschen. Der Zugang zu palliativer Versorgung von COVID-19-Patientinnen und -Patienten muss unabhängig vom Ort der Behandlung (Heim, Spital oder zu Hause) gewährleistet sein. Ebenso muss die psychiatrische, psychotherapeutische und psychologische Betreuung gestärkt werden, um den akuten Bedarf zu bewältigen und psychische Langzeitfolgen der Pandemie zu lindern. Zur Stärkung der Grundversorgung gehört auch der Ausbau der Telemedizin.

\section{Schutz vulnerabler Personengruppen}

Der Schutz, das Wohlergehen und der Erhalt der Lebensqualität von vulnerablen Personen ist eine gemeinschaftliche Aufgabe und dadurch auch grundlegende Pflicht aller Akteurinnen und Akteure im Gesundheits- und Sozialwesen. Die COVID-19-Pandemie hat unterschiedliche Personengruppen besonders verletzlich gemacht. Diese Verletzlichkeit kann durch das Lebensalter (z.B. Kinder, Betagte), soziale Umstände (Jugendliche im Schulalter, Alleinerziehende, Heimbewohnerinnen und -bewohner), die gesundheitliche Situation (z.B. Risikogruppen) oder den Arbeitsort (Gesundheits- und Sozialwesen) verstärkt werden. Auf die Wahrung der Persönlichkeitsrechte von Bewohnerinnen und Bewohnern von Pflegeheimen und weiteren Wohneinrichtungen ist unbedingt $\mathrm{zu}$ achten. Dies schliesst den garantierten Zugang von essential visitors ein, d.h. pflegenden Angehörigen, Beiständen und Fachpersonen (z.B. Hausärztinnen und -ärzte, Seelsorgerinnen und -sorger etc.). Das Gesundheitspersonal bedarf effektiver Schutzmassnahmen zum Erhalt der physischen und psychischen Gesundheit. Es muss mit allen Mitteln verhindert werden, dass Mitarbeitende

\section{Das Gesundheitspersonal bedarf effektiver} Schutzmassnahmen zum Erhalt der physischen und psychischen Gesundheit.

mit bekanntem positivem Befund Kontakt mit Patientinnen, Patienten, Bewohnerinnen und Bewohnern haben. Zudem haben erkrankte Mitarbeitende Anrecht auf eine angemessene Zeit der Genesung. Für ethische Fragestellungen im Umgang mit vulnerablen Personen sollten flächendeckend niederschwellige Strukturen der ethischen Unterstützung zur Verfügung stehen.

\section{Transparente Triage- und Verlegungskriterien sowie Koordination}

Ressourcen müssen lokal und national optimal koordiniert werden, um Triagesituationen für alle Patientinnen und Patienten im Kontext der COVID-19-Pandemie $\mathrm{zu}$ verhindern. Eine rein vorsorgliche «stille» (oder implizite) Triage gewisser Personengruppen, z.B. aufgrund des Alters oder des Wohnorts Pflegeheim, ist ethisch nicht zulässig. Zeichnet sich die Notwendigkeit einer Triage ab, müssen die Kriterien transparent sein, etablierte Richtlinien [1] berücksichtigen und den Willen der betroffenen Person einbeziehen. Wird auf lokaler Ebene eine Triage notwendig, bevor eine natio- 
nale Triage und Koordination etabliert ist - z.B. wegen fehlender lokaler Kapazitäten und Verlegungsoptionen -, muss auch die lokale Triage transparent sein und sich an den Richtlinien der SAMW/SGI [1] orientieren. Für ethische Fragestellungen im Kontext von Triageentscheidungen sollten Strukturen der ethischen Unterstützung vor Ort verfügbar sein.

\section{Nachhaltige Finanzierung}

Nebst der Grundversorgung leisten auch Spitäler und Kliniken einen unverzichtbaren Beitrag zur Bewältigung der Pandemie. Die Pflicht zu Bereitschaftsleistungen während der COVID-19-Pandemie hat bei vielen Spitälern zu grossen, bisher nicht oder ungenügend gedeckten Ertragsausfällen geführt. Fragen der fairen Finanzierung resp. der staatlichen Refinanzierung unterfinanzierter Spitäler sollten mit allen Beteiligten explizit und rasch geklärt werden. Die aktuelle CoronaPandemie darf keine nachteiligen Auswirkungen auf die Spitallisten-Verträge haben. Ebenso sollte während der Pandemie die Fallzahlenerhebung für die anstehende Vergabe der Spitalleistungsaufträge sistiert werden, mit Ausnahme der Fallzahlenerhebungen durch den koordinierten Sanitätsdienst. Neben der stationären muss aber auch die ambulante Versorgung in materieller, finanzieller und personeller Hinsicht gleichbehandelt und gefördert werden, um die schweren Auswirkungen der Krise besser zu bewältigen. Durch eine transparente, koordinierte Verteilung von Patientinnen und Patienten können lokale Triagesituationen verhindert werden, was die Gesundheitsversorgung für die ganze Bevölkerung sichert.

\section{Mitautorinnen und Mitautoren}

Bernice Elger, Prof. Dr. med.; Isabelle Karzig, MScN, MAE; Diana Meier-Allmendinger, Dr. med., lic. theol.; Andrea Moser, MScN; Reto Stocker, Prof. Dr. med.; Manuel Trachsel, PD Dr. med. Dr. phil.; Tatjana Weidmann-Hügle, MSc, MA

\section{Das Wichtigste in Kürze}

Settimio Monteverde Universität Zürich Institut für Biomedizinische Ethik und Medizingeschichte

Winterthurerstrasse 30

CH-8006 Zürich

Tel. 0446344081

settimio.monteverde[at] uzh.ch schaft vor grosse Herausforderungen. Medizinethikerinnen und -ethiker der Schweiz formulieren vier Postulate, die den solidarischen und verantwortungsvollen Umgang mit der Krise sicherstellen sollen.

- Sie fordern: die Stärkung der Grundversorgung, den Schutz vulnerabler Personengruppen, transparente Triage- und Verlegungskriterien sowie Koordination und eine nachhaltige (Re-)Finanzierung der medizinischen Leistungen.

\section{Folgende Personen unterstützen diesen Appell (in alphabetischer Reihenfolge)}

- Andrea Büchler, Prof. Dr. iur. Dr. h.c., Präsidentin Nationale Ethikkommission im Bereich der Humanmedizin NEK

- Thomas Gruberski, Dr. iur., und Sibylle Ackermann, lic. theol., Dipl.-Biol., Ressort Ethik, Schweizerische Akademie der Medizinischen Wissenschaften

- Thomas Heiniger, Dr. iur., Präsident Spitex Schweiz

- Annina Hess-Cabalzar, MA Psychotherapie, und David Nadal, Prof. Dr. med., Präsidium Akademie Menschenmedizin

- Susanne Hochuli, Präsidentin Schweizerische Stiftung SPO Patientenorganisation

- Samia Hurst, Prof. Dr. med., Swiss National COVID-19 Science Task Force, Leiterin Expertengruppe Ethische, rechtliche und soziale Fragen

- Isabelle Moret, Präsidentin H+ Die Spitäler der Schweiz

- Bianca Schaffert, MScN, Präsidentin Ethikkommission Schweizerischer Berufsverband der Pflegefachfrauen und Pflegefachmänner

- Johanna Sommer, Prof. Dr. med. (FMH AIM), Vorsteherin Institut Hausarztmedizin, Medizinische Fakultät, Universität Genf

- Franziska Sprecher, Prof. Dr. iur., Professorin für Gesundheitsrecht, Universität Bern

- Laurent Wehrli, Präsident CURAVIVA Schweiz, und Markus Leser, Dr. phil., Leiter Fachbereich Menschen im Alter CURAVIVA Schweiz

Literatur

1 www.samw.ch/dam/jcr:14cb0e53-eedb-4089-96dd-b49a1b388994/ richtlinien v3-1 samw triage intensivmedizinische massnahmen_ressourcenknappheit_20201217.pdf

2 www.sgi-ssmi.ch/files/Dateiverwaltung/COVID 19/Stellungnahmen\%20SGI/IMSGCVCM_Stellungnahme_COVID-19_201217 DE_06.pdf

3 www.nek-cne.admin.ch/de/ueber-uns/news/news-details/nek-veroeffentlicht-stellungnahme-zum-schutz-der-persoenlichkeit-ininstitutionen-der-langzeitpfleg/

4 https://sciencetaskforce.ch/expertengruppe-ethics-legal-social/

5 www.sbk.ch/aktuell/news-single?tx_news_pi1\%5Bnews\%5D=529\& cHash $=945$ dcacc3bc32ec49159514feea $8 d 88 b$

6 www.sbk.ch/files/sbk/Aktuell/COVID 19/2020 05 08 Eth Herausforderungen_SARS-CoV2_def_d.pdf

7 www.sbk.ch/files/sbk/pflegethemen/docs/SBK-ASI Ethik Standpunkt_Isolation.pdf zur-zeit-vom-13-november-2020

9 https://saez.ch/article/doi/saez.2020.19037

\section{Bildnachweis}

(c) Ristinose | Dreamstime.com, Symbolbild
8 www.dialog-ethik.ch/ueber-uns/aktuell/kommentar- 